\title{
AFECTACIÓN DE LOS DERECHOS ECONOMICOS, SOCIALES Y CULTURALES POR LA IMPLEMENTACIÓN DEL SISTEMA DE TRANSPORTE MASIVO, TRANSCARIBE, EN EL BARRIO DANIEL LEMAITRE DE CARTAGENA-COLOMBIA
}

Affection of economic, social and cultural rights due to the implementation of

"Transcaribe" massive transportation system in Daniel Lemaitre neighborhood

of Cartagena - Colombia.

Marta Lucía García González ${ }^{1}$

Mario Armando Echeverria Acuña ${ }^{2}$

\section{Recibido: 29 de septiembre 2019 - Aceptado: 10 de diciembre de 2019}

\section{RESUMEN}

El objetivo de esta investigación consiste en explorar de manera crítica, la implementación del Sistema Integrado de Transporte Masivo, en la ciudad de Cartagena como estrategia gubernamental para mejorar la movilidad urbana y como esta acción trastoca los derechos humanos de un sector vulnerable de la ciudad, por ello, la unidad de análisis es examinar de qué manera podría impactar la implementación de la política pública del Sistema Integrado de Transporte Masivo TRANSCARIBE los derechos económicos, sociales y culturales de los propietarios y conductores de colectivos en la ruta Daniel Lemaitre en la ciudad de Cartagena.

Palabras clave: Política pública; sistema integrado de transporte masivo "TRANSCARIBE"; ruta de transporte público; Daniel Lemaitre-San Francisco; movilidad urbana; transporte informal.

\footnotetext{
1 Abogada, Magister en Derecho Administrativo (C) de la Universidad Libre - Sede Cartagena. Orcid: https://orcid.org/0000-0001-8267-0785 E-mail: martagarcia2324@gmail.com

2 Abogado, Especialista en Derecho Constitucional y Administrativo, Magister en Derecho, Candidato a Doctor en Ciencias Sociales, Docente investigador del Programa de Derecho de la Universidad Libre Sede Cartagena. Orcid: https://orcid.org/0000-0003-4831-6277 E-mail: mario.echeverria@unilibrectg.edu.co
} 
Marta Lucía García González y Mario Armando Echeverria Acuña

\section{ABSTRACT}

The main purpose of this research is to critically explore the implementation of the Integrated Mass Transportation System in the city of Cartagena as a government strategy to improve urban mobility and how these actions upset the human rights of a vulnerable sector of the city. Therefore, the unit of analysis is to examine how the implementation of public policy of the Integrated System of Mass Transportation TRANSCARIBE could impact the economic, social and cultural rights of the owners and drivers of buses on the Daniel Lemaitre route in the city of Cartagena.

Keywords: Public policy; integrated mass transportation system "TRANSCARIBE"; Daniel Lemaitre-San Francisco; public transportation's route; urban mobility; informal transportation.

\section{INTRODUCCIÓN}

Los Sistemas Integrados de Transporte Masivo (SITM) forman parte de la política para mejorar el servicio de transporte público urbano de pasajeros contemplada en el Conpes 3167 de 2002, la cual fue orientada a otras ciudades de este país como Cali, Bucaramanga, Barranquilla, Pereira y Cartagena, teniendo como referente al Sistema de Transmilenio, considerado como proyecto hito urbano, el cual fue implementado en la Ciudad de Bogotá en el año 2000, con la finalidad de mejorar la movilidad. Siguiendo a Hurtado et al (2011), dentro de este tipo de megaproyectos de infraestructuras, se generan dudas complejas por los distintos actores e intereses que intervienen en el sector público y privado. Por ello, el problema de investigación se centra, en que según el Decreto No 0667 de 2008, emanado del Departamento Administrativo de Tránsito y Transporte (DATT) se reestructuró el recorrido que hacían los buses de la Ruta No 18, Daniel Lemaitre hacia el centro de la ciudad de Cartagena y viceversa, que como característica este tenía como recorrido salida del barrio San Francisco, Calle 76, Carrera 17 - En el barrio Daniel Lemaitre- Paseo Bolívar, Puente las Palmas, Avenida del Lago, Puente Román, Calle del Pedregal, Calle 30, Carrera 17, Paseo de Bolívar, Calle 76, Terminal; además debía ser prestado este servicio público con buses de una longitud de $11+000$ kilómetros en un tiempo de recorrido de 121 minutos en una jornada desde las 5:00 am hasta las 21 horas con 59 minutos. Esta ruta fue entregada a las empresas RENACIENTE (Con 19 buses), RODRÍGUEZ TORICES (Con 18 buses), ETUL (Con 6 buses), Cootransbol que luego se convirtió en VEHITRANS (Con 6 buses), FLOTA DE LUJO (Con 4 buses), MEDIA LUNA (Con 4 buses) y PEMAPE (Con 3 buses) para un total de sesenta (60) buses urbanos que hacían cubrimiento de la ruta.

También se reestructuró la Ruta No 19, Daniel Lemaitre hacia el mercado de Bazurto y viceversa en la ciudad de Cartagena, que venía siendo regulado por el Decreto No 243 de 
1987 emanado de la Alcaldía Mayor de Cartagena, que tenía como característica un recorrido con salida del barrio San Francisco, Calle 76, Carrera 17 - En el barrio Daniel Lemaitre- Calle 30, Avenida Pedro de Heredia, Mercado, Avenida Jacobo del Valle Recuero, Carrera 17, Calle 76, Terminal, Barrio San Francisco; además debía ser prestado este servicio público con buses de una longitud de $18+000$ kilómetros en un tiempo de recorrido de 105 minutos en una jornada desde las 5:00 am hasta las 21 horas. Esta ruta fue entregada a las empresas RENACIENTE (Con 9 buses), RODRÍGUEZ TORICES (Con 10 buses), ETUL (Con 4 buses), Cootransbol que luego se convirtió en VHITRANS (Con 4 buses), FLOTA DE LUJO (Con 4 buses), MEDIA LUNA (Con 3 buses) y PEMAPE (Con tres buses) para un total de Treinta y siete (37) buses que cubrían dicha ruta.

La anterior restructuración seria transitoria teniendo en cuenta que se implementaría el sistema masivo de transporte integrado al que llamaron TRANSCARIBE, pero la realidad social (Berger et al: 1996) que, desde hace muchos años, viven los habitantes de barrios como Paseo Bolívar, Daniel Lemaitre, San Francisco y 7 de Agosto aledaños a la falda de la Popa, es que no cuentan con un servicio público de transporte urbano de pasajeros, porque el parque automotor de buses urbanos fue chatarrizado dando cumplimiento a la Ley 688 de 2001 que creó el Fondo Nacional para la Reposición del parque automotor del servicio público de transporte terrestre, que en el artículo 21 reguló lo concerniente a la desintegración física de automotores, indicando que los vehículos que cumplieran con su ciclo de vida útil debían pasar a un proceso de desintegración física. Este proceso de chatarrización debió ser reglamentado por el Ministerio de Transporte y controlado por las autoridades competentes en Cartagena.

Es así como la necesidad de movilidad de los ciudadanos que viven en estos sectores, fue suplida por un grupo de particulares que prestan el servicio de transporte por medio de carros camperos que comúnmente han sido llamados colectivos, desbordando un crecimiento del transporte público informal y forjando un transporte supuestamente más rápido y económico, fenómeno que generó aceptación en el tiempo y en condiciones permitidas por la administración pública, tal como se reconoce en la acción de tutela accionada por German Pacheco Cardales y otros, contra la Alcaldía Mayor de Cartagena, el DATT y TRANSCARIBE SITM, impetrada ante el Juzgado Tercero Penal Municipal para adolescentes con función de garantías en el año 2017, por la presunta violación a los derechos fundamentales de petición, debido proceso, mínimo vital e igualdad.

El concepto de movilidad urbana "comprende todos los elementos asociados al desplazamiento de personas y bienes a través del espacio urbano. Las ciudades son espacios dinámicos y en este sentido la movilidad posibilita el flujo para que ésta pueda operar adecuadamente" (Saldarriaga, 2007). La ciudad define un modelo de cómo quiere 
Marta Lucía García González y Mario Armando Echeverria Acuña

verse a través de estrategias que tengan que ver con el desarrollo urbano, la movilidad urbana califica entre ellas como estrategia que se debe a la circulación y el transporte público, de esta manera permite el desplazamiento en la ciudad y atiende necesidades de la población en este contexto. (Correa et al: 2014.)

Para mejorar la movilidad urbana de la ciudad de Cartagena, el Sistema Integral Masivo de Transporte TRANSCARIBE, empezó a circular a partir del 17 de noviembre de 2015, bajo el mandato del ex alcalde, Dionisio Vélez Trujillo, quien logró sacar adelante el macroproyecto una década después, logrando así rodar los primeros 7 buses en la etapa pedagógica (Acieri, 2015) sin embargo después de cinco años en los sectores como la ruta Daniel Lemaitre, que está planeado como recorrido complementario o auxiliar en la cual operaran vehículos de tipo busetón con capacidad para 50 pasajeros, pero que aún no se implementa el servicio y que de ponerse en práctica tendría como resultado, un impacto que repercutiría en lo social, en lo urbano, en la actividad económica, en la cultura del transporte informal llamado colectivos, por lo que posiblemente entrarán en riesgo muchas familias de propietarios y conductores de los vehículos mencionados, que dependen y derivan su sustento de la actividad de transportistas que desempeñan desde este medio de transporte, y quienes están preocupadas por el futuro incierto que se vislumbra con la entrada en funcionamiento de la ruta de Transcaribe por Daniel Lemaitre, teniendo en cuenta la actividad económica ejercida por estos, derivada del servicio de transporte informal como actividad practicada por más de 25 años y que ha brindado un servicio a habitantes de comunidades que quedaron olvidadas por la Administración Distrital.

En la actualidad existe una gran tensión entre el Distrito de Cartagena y el gremio de transportadores informales que forma parte de la Cooperativa Multiactiva de Transporte Colectivo Comutrascol, quienes han generado acciones de tutelas además de protestas para exigir su derecho al trabajo y tratar de llegar a un acuerdo que no vulnere sus derechos, teniendo en cuenta que son muchas familias que dependen de la actividad informal de transporte, por ello la pregunta que orienta esta investigación es: ¿De qué manera podría impactar la implementación de la política pública del Sistema Integrado de Transporte Masivo TRANSCARIBE los derechos económicos, sociales y culturales de los propietarios y conductores de colectivos en la ruta Daniel Lemaitre, en la ciudad de Cartagena?

Esta investigación se justifica porque es relevante ya que el transporte masivo Transcaribe en varios sectores de la ciudad de Cartagena se puso en marcha, sin embargo, en otras zonas faltó, como en la ruta que cubre al barrio Daniel Lemaitre. Esta acción si es necesaria porque la implementación del sistema Masivo de Transcaribe, influye en una población que utiliza un transporte informal, pero que su ejecución afecta sus derechos porque dependen de su actividad económica, ya que la misma es de interés para la comunidad pues además 
del conflicto social por la asignación del Transcaribe, se genera un debate jurídico por la posible violación del principio de la confianza legítima por omisión, de la administración Distrital de Cartagena.

El objetivo general fue examinar de qué manera podría impactar la implementación de la política pública del Sistema Integrado de Transporte Masivo TRANSCARIBE los derechos económicos, sociales y culturales de los propietarios y conductores de colectivos en la ruta Daniel Lemaitre, en la ciudad de Cartagena y los objetivos específicos, fueron: Realizar un análisis Normativo y Doctrinal sobre la política pública del Sistema Integrado de Transporte Masivo TRANSCARIBE en Cartagena de Indias y Realizar un sondeo estadístico con quienes habitan en la ruta trazada para Transcaribe en el barrio Daniel Lemaitre de la ciudad de Cartagena para indagar de qué manera podría impactar la implementación de la política pública del Sistema Integrado de Transporte Masivo TRANSCARIBE los derechos, económicos, sociales y culturales de los propietarios y conductores de colectivos.

La hipótesis que se plantea, es que es muy probable que la implementación de la política pública del sistema integral de transporte masivo TRANSCARIBE en la ruta Daniel Lemaitre podría vulnerar los derechos económicos, sociales y culturales de los propietarios y conductores de los colectivos que cubren dicha ruta en la ciudad de Cartagena, pues aunque este sea un transporte informal les cabe la aplicación del principio de la confianza legítima y es factible que estas personas presenten tutelas contra el Distrito de Cartagena, para reclamar los derechos fundamentales a la vida y al trabajo digno, que suele ser vulnerados cuando el Estado hace estos procedimientos y que un juez puede garantizar a través de la acción de tutela, por ejemplo, porque permite la protección de los ciudadanos de cambios bruscos e intempestivos efectuados por las autoridades estatales. La Corte Constitucional de Colombia en Sentencia T - 424 de 2017, estableció los presupuestos que deben acreditarse para su materialización y para que no prospere la invocación del Principio de la Confianza Legítima, es que la medida de recuperación de la Ruta Daniel Lemaitre por parte de TRANSCARIBE debe atender un fin constitucional, que no es el caso en esta investigación.

\section{El dispositivo metodológico}

Metodológicamente esta investigación socio-jurídica es de corte cuantitativa (Ramírez,2001) porque se analiza la eficacia de la implementación de la política pública del Sistema Integrado de Transporte Masivo TRANSCARIBE en la Ruta Daniel Lemaitre en Cartagena de Indias, mediante la realización un sondeo estadístico aplicado a quienes habitan en la ruta trazada para Transcaribe en el barrio Daniel Lemaitre de la ciudad de Cartagena para indagar de qué manera podría impactar la implementación de la política pública del Sistema Integrado de Transporte Masivo TRANSCARIBE los derechos 
Marta Lucía García González y Mario Armando Echeverria Acuña

económicos, sociales y culturales de los propietarios y conductores de colectivos en la ruta Daniel Lemaitre, en la ciudad de Cartagena. Los métodos cuantitativos contribuyen a establecer, en longitudes, las condiciones iníciales de los beneficiarios y el cambio resultante por su participación en un programa.

\section{Resultados}

Para la realización de esta investigación, según lo dicho por Herreño et al (2008) se parte del supuesto teórico según el cual la gobernabilidad es un proceso que determina la capacidad del Estado para canalizar y responder a los intereses y demandas de la sociedad de forma eficiente y eficaz, en una dinámica de interacción continua, mediante mecanismos de diálogo y participación, para alcanzar el bienestar social dentro de un marco institucional adecuado, que refleja los valores y las reglas que la sociedad ha impuesto, por esto la población objeto del sondeo estadístico de la investigación es la que habita en los barrios Paseo Bolívar, Daniel Lemaitre, San Francisco y Siete de Agosto que forman parte de la unidad comunera de gobierno No 3 en la Localidad No 1, Histórica y del Caribe del Distrito de Cartagena de Indias que cuenta con Trescientos ochenta y ocho mil (388) cuatrocientos noventa y ocho (498) habitantes. La muestra objeto de estudio fue definida a partir de la ruta de Daniel Lemaitre del SITM Transcaribe y se escogieron al azar 33 personas que habitan cerca de la ruta que son afectadas por no tener servicio público de transporte urbano de pasajeros y que utilizan los colectivos afiliados a la Cooperativa Multiactiva de Transporte Colectivo, Comutrascol.

\subsection{Los Instrumentos Aplicados}

En el sondeo estadístico se aplicaron encuestas sociales de opinión que pretende establecer las percepciones de los habitantes de los barrios Paseo Bolívar, Daniel Lemaitre, San Francisco y Siete de Agosto sobre de qué manera podría impactar la implementación de la política pública del Sistema Integrado de Transporte Masivo TRANSCARIBE los derechos, económicos, sociales y culturales de los propietarios y conductores de colectivos integrantes de la Cooperativa Multiactiva de Transporte Colectivo, Comutrascol. Para la encuesta estructurada se elaboró un cuestionario de preguntas cerradas donde el entrevistado solo responde sí o no, este es el análisis de las encuestas:

Grafica No 1: ¿Tiene usted conocimiento de los beneficios de la política pública del sistema integrado de transporte masivo, Transcaribe?

\begin{tabular}{|l|l|l|}
\hline OPCIÓN & FRECUENCIA & PORCENTAJE \\
\hline $\mathrm{Si}$ & 20 & $61 \%$ \\
\hline No & 13 & $39 \%$ \\
\hline TOTAL & 33 & $\mathbf{1 0 0 \%}$ \\
\hline
\end{tabular}

Fuente: Encuestas realizada por el investigador. 
Los encuestados, quienes habitan cerca de la ruta que son afectadas por no tener servicio público de transporte urbano de pasajeros y que utilizan los colectivos afiliados a la Cooperativa Multiactiva de Transporte Colectivo, Comutrascol en Cartagena de Indias (Colombia), dijeron que tener conocimiento de los beneficios de la política pública del sistema integrado de transporte masivo, Transcaribe; Si en un $61 \%$ y que No en un $39 \%$.

Grafica No 2: ¿Conoce usted si la ruta de Daniel Lemaitre-San Francisco hace parte del sistema Integrado de transporte masivo de pasajeros, Transcaribe?

\begin{tabular}{|l|l|l|}
\hline OPCIÓN & FRECUENCIA & PORCENTAJE \\
\hline $\mathrm{Si}$ & 19 & $58 \%$ \\
\hline No & 14 & $42 \%$ \\
\hline TOTAL & 33 & $\mathbf{1 0 0 \%}$ \\
\hline
\end{tabular}

Fuente: Encuestas realizada por el investigador.

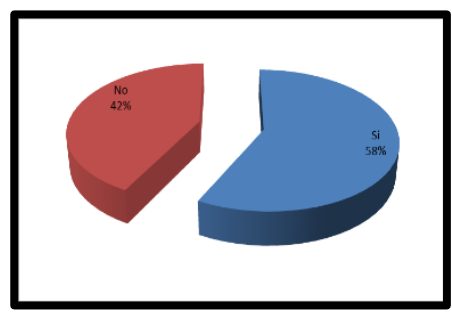

Los encuestados, quienes habitan cerca de la ruta que son afectadas por no tener servicio público de transporte urbano de pasajeros y que utilizan los colectivos afiliados a la Cooperativa Multiactiva de Transporte Colectivo, Comutrascol en Cartagena de Indias (Colombia), conocen de si la ruta de Daniel Lemaitre-San Francisco hace parte del sistema Integrado de transporte masivo de pasajeros, Transcaribe, Si en un $58 \%$ y que No en un $42 \%$.

Grafica No 3: ¿Fueron ustedes convocados en la estructuración de la política pública del sistema integrado de transporte masivo de pasajeros Transcaribe para la ruta Daniel Lemaitre-San Francisco?

\begin{tabular}{|l|l|l|}
\hline OPCIÓN & FRECUENCIA & PORCENTAJE \\
\hline $\mathrm{Si}$ & 13 & $39 \%$ \\
\hline No & 20 & $61 \%$ \\
\hline TOTAL & 33 & $100 \%$ \\
\hline
\end{tabular}

Fuente: Encuestas realizada por el investigador.

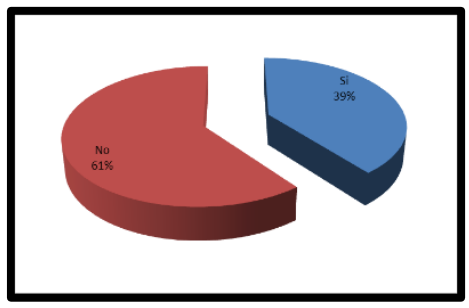

Los encuestados, quienes habitan cerca de la ruta que son afectadas por no tener servicio público de transporte urbano de pasajeros y que utilizan los colectivos afiliados a la Cooperativa Multiactiva de Transporte Colectivo, Comutrascol en Cartagena de Indias (Colombia), fueron convocados en la estructuración de la política pública del sistema integrado de transporte masivo de pasajeros Transcaribe para la ruta Daniel Lemaitre-San Francisco, Si en un $39 \%$ y que No en un $61 \%$. 
Marta Lucía García González y Mario Armando Echeverria Acuña

Grafica No 4: ¿Considera usted que se verá afectado el derecho al trabajo y al mínimo vital de los propietarios y conductores de colectivos y camperos que actualmente cubren la ruta Daniel Lemaitre-San Francisco con la implementación del sistema Integrado de transporte masivo de pasajeros, Transcaribe?

\begin{tabular}{|l|l|l|}
\hline OPCIÓN & FRECUENCIA & PORCENTAJE \\
\hline $\mathrm{Si}$ & 30 & $91 \%$ \\
\hline No & 3 & $9 \%$ \\
\hline TOTAL & 33 & $\mathbf{1 0 0 \%}$ \\
\hline
\end{tabular}

Fuente: Encuestas realizada por el investigador.

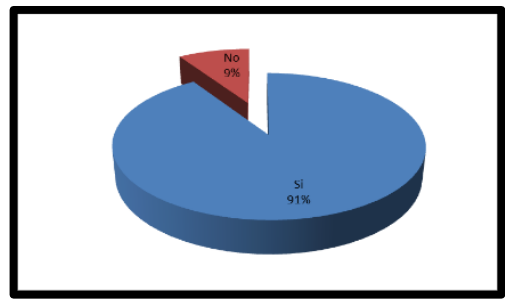

Los encuestados, quienes habitan cerca de la ruta que son afectadas por no tener servicio público de transporte urbano de pasajeros y que utilizan los colectivos afiliados a la Cooperativa Multiactiva de Transporte Colectivo, Comutrascol en Cartagena de Indias (Colombia), consideran que se verá afectado el derecho al trabajo y al mínimo vital de los propietarios y conductores de colectivos y camperos que actualmente cubren la ruta Daniel Lemaitre-San Francisco con la implementación del sistema Integrado de transporte masivo de pasajeros, Transcaribe; Si en un $91 \%$ y que No en un $9 \%$.

Grafica No 5: ¿Conoce usted si el servicio informal de transporte de colectivos y camperos cubre actualmente la necesidad de transporte de las personas que residen en los barrios Paseo Bolívar, Daniel Lemaitre y San Francisco?

\begin{tabular}{|l|l|l|}
\hline OPCIÓN & FRECUENCIA & PORCENTAJE \\
\hline $\mathrm{Si}$ & 21 & $64 \%$ \\
\hline No & 12 & $36 \%$ \\
\hline TOTAL & 33 & $\mathbf{1 0 0 \%}$ \\
\hline
\end{tabular}

Fuente: Encuestas realizada por el investigador.

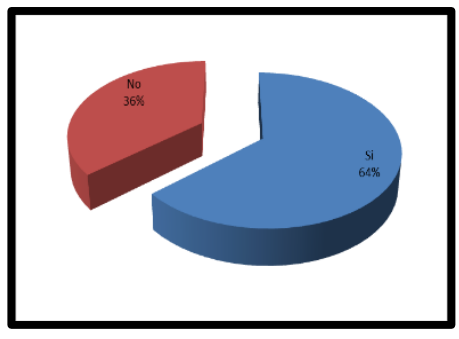

Los encuestados, quienes habitan cerca de la ruta que son afectadas por no tener servicio público de transporte urbano de pasajeros y que utilizan los colectivos afiliados a la Cooperativa Multiactiva de Transporte Colectivo, Comutrascol en Cartagena de Indias (Colombia), conocen si el servicio informal de transporte de colectivos y camperos cubre actualmente la necesidad de transporte de las personas que residen en los barrios Paseo Bolívar, Daniel Lemaitre y San Francisco; Si en un 64\% y que No en un $36 \%$.

Grafica No 6: ¿Ha sido usted invitado a capacitaciones o le han socializado la implementación del sistema Integrado de transporte masivo de pasajeros, Transcaribe en la ruta de Daniel Lemaitre-San Francisco, por parte de la gerencia de Transcaribe o la Alcaldía de Cartagena? 


\begin{tabular}{|l|l|l|}
\hline OPCIÓN & FRECUENCIA & PORCENTAJE \\
\hline $\mathrm{Si}$ & 2 & $6 \%$ \\
\hline No & 31 & $94 \%$ \\
\hline TOTAL & 33 & $\mathbf{1 0 0 \%}$ \\
\hline
\end{tabular}

Fuente: Encuestas realizada por el investigador.

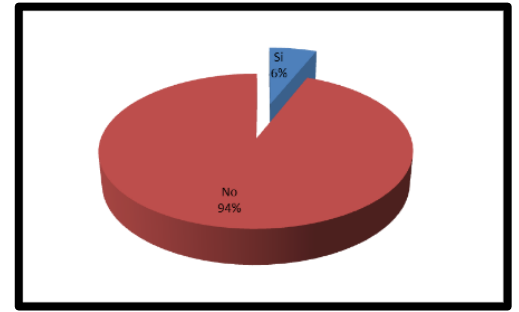

Los encuestados, quienes habitan cerca de la ruta que son afectadas por no tener servicio público de transporte urbano de pasajeros y que utilizan los colectivos afiliados a la Cooperativa Multiactiva de Transporte Colectivo, Comutrascol en Cartagena de Indias (Colombia), ha sido invitado a capacitaciones o le han socializado la implementación del sistema Integrado de transporte masivo de pasajeros, Transcaribe en la ruta de Daniel Lemaitre-San Francisco, por parte de la gerencia de Transcaribe o la Alcaldía de Cartagena; Si en un $6 \%$ y que No en un $94 \%$.

Grafica No 7: ¿Considera usted que la implementación del sistema Integrado de transporte masivo de pasajeros, Transcaribe en la Ruta Daniel Lemaitre-San Francisco perjudica económica o patrimonialmente a los propietarios y conductores de colectivos y camperos que actualmente cubren la ruta?

\begin{tabular}{|l|l|l|}
\hline OPCIÓN & FRECUENCIA & PORCENTAJE \\
\hline $\mathrm{Si}$ & 21 & $64 \%$ \\
\hline No & 12 & $36 \%$ \\
\hline TOTAL & 33 & $\mathbf{1 0 0 \%}$ \\
\hline
\end{tabular}

Fuente: Encuestas realizada por el investigador.

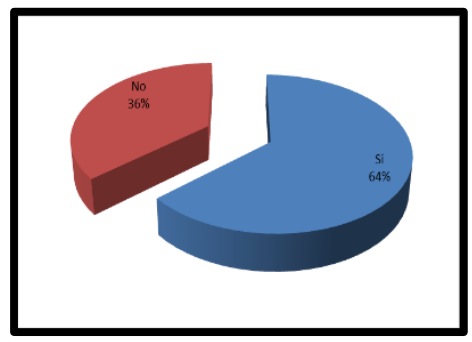

Los encuestados, quienes habitan cerca de la ruta que son afectadas por no tener servicio público de transporte urbano de pasajeros y que utilizan los colectivos afiliados a la Cooperativa Multiactiva de Transporte Colectivo, Comutrascol en Cartagena de Indias (Colombia),considera que la implementación del sistema Integrado de transporte masivo de pasajeros, Transcaribe en la Ruta Daniel Lemaitre-San Francisco perjudica económicamente o patrimonialmente a los propietarios y conductores de colectivos y camperos que actualmente cubren la ruta, Si en un $64 \%$ y que No en un $36 \%$.

Grafica No 8: ¿Cree usted que deben ser reparados los propietarios y conductores de colectivos y camperos por ser trabajadores del servicio público de transporte informal, luego de la implementación del sistema de transporte masivo Transcaribe en la ruta Daniel Lemaitre-San Francisco, por parte de la gerencia de Transcaribe o el Distrito de Cartagena? 


\begin{tabular}{|l|l|l|}
\hline OPCIÓN & FRECUENCIA & PORCENTAJE \\
\hline $\mathrm{Si}$ & 23 & $70 \%$ \\
\hline No & 10 & $30 \%$ \\
\hline TOTAL & 33 & $\mathbf{1 0 0 \%}$ \\
\hline
\end{tabular}

Fuente: Encuestas realizada por el investigador.

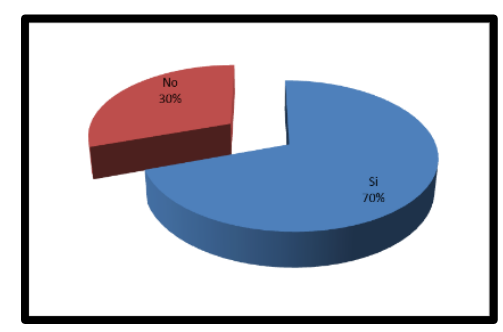

Los encuestados, quienes habitan cerca de la ruta que son afectadas por no tener servicio público de transporte urbano de pasajeros y que utilizan los colectivos afiliados a la Cooperativa Multiactiva de Transporte Colectivo, Comutrascol en Cartagena de Indias (Colombia), creen que deben ser reparados los propietarios y conductores de colectivos y camperos por ser trabajadores del servicio público de transporte informal, luego de la implementación del sistema de transporte masivo Transcaribe en la ruta Daniel LemaitreSan Francisco, por parte de la gerencia de Transcaribe o el Distrito de Cartagena; Si en un $70 \%$ y que No en un $30 \%$.

Grafica No 9: ¿Cree usted que los propietarios y conductores de colectivos y camperos por ser trabajadores del servicio público de transporte informal deben ser incluidos en algún estudio socioeconómico por parte del distrito que evalué los daños que se deriven de la implementación del sistema integrado masivo Transcaribe en la ruta Daniel Lemaitre-San Francisco en los usuarios y trabajadores que dependen del transporte informal?

\begin{tabular}{|l|l|l|}
\hline OPCIÓN & FRECUENCIA & PORCENTAJE \\
\hline $\mathrm{Si}$ & 29 & $88 \%$ \\
\hline No & 4 & $12 \%$ \\
\hline TOTAL & 33 & $\mathbf{1 0 0 \%}$ \\
\hline
\end{tabular}

Fuente: Encuestas realizada por el investigador.

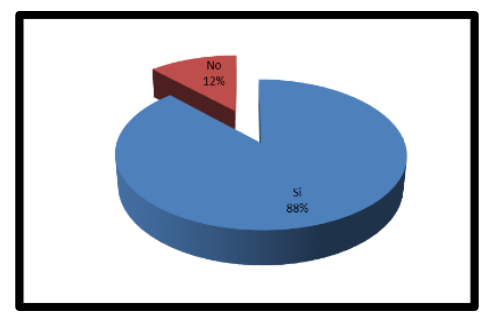

Los encuestados, quienes habitan cerca de la ruta que son afectadas por no tener servicio público de transporte urbano de pasajeros y que utilizan los colectivos afiliados a la Cooperativa Multiactiva de Transporte Colectivo, Comutrascol en Cartagena de Indias (Colombia), creen que los propietarios y conductores de colectivos y camperos por ser trabajadores del servicio público de transporte informal deben ser incluidos en algún estudio socioeconómico por parte del distrito que evalué los daños que se deriven de la implementación del sistema integrado masivo Transcaribe en la ruta Daniel Lemaitre-San Francisco en los usuarios y trabajadores que dependen del transporte informal; $\mathrm{Si}$ en un $88 \%$ y que No en un $12 \%$.

Grafica No 10: ¿Considera usted que la ciudad de Cartagena se ha beneficiado con la implementación de la política pública del sistema de transporte masivo Transcaribe? 


\begin{tabular}{|l|l|l|}
\hline OPCIÓN & FRECUENCIA & PORCENTAJE \\
\hline $\mathrm{Si}$ & 30 & $91 \%$ \\
\hline No & 3 & $9 \%$ \\
\hline TOTAL & 33 & $\mathbf{1 0 0 \%}$ \\
\hline
\end{tabular}

Fuente: Encuestas realizada por el investigador.

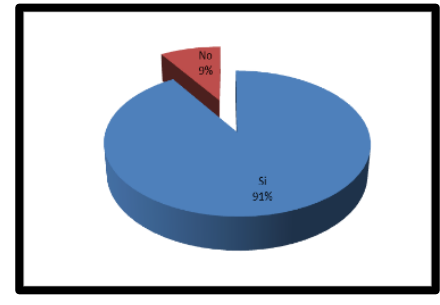

Los encuestados, quienes habitan cerca de la ruta que son afectadas por no tener servicio público de transporte urbano de pasajeros y que utilizan los colectivos afiliados a la Cooperativa Multiactiva de Transporte Colectivo, Comutrascol en Cartagena de Indias (Colombia), considera que la ciudad de Cartagena se ha beneficiado con la implementación de la política pública del sistema de transporte masivo Transcaribe, Si en un $91 \%$ y que No en un $9 \%$.

\section{Discusión}

En este acápite haremos un análisis teórico al tema que se examina en relación con los resultados obtenidos en las encuestas realizadas en quienes habitan cerca de la ruta que son afectadas por no tener servicio público de transporte urbano de pasajeros y que utilizan los colectivos afiliados a la Cooperativa Multiactiva de Transporte Colectivo, Comutrascol en Cartagena de Indias (Colombia)

\subsection{Influencia de Revolución francesa en los servicios públicos}

Brevemente En Francia en los años 1700, lidiaban con el antiguo régimen, bajo la monarquía de los Borbones, el cual se caracterizaba por el absolutismo real, "Princeps legibus solutus: el rey no está sujeto al derecho", contexto en el que primaba la desigualdad social, política y económica, donde la concepción de los servicios públicos se desconocía, sin embargo con la revolución francesa en 1789, el panorama se transforma a través de los ideales filosóficos de Rousseau, Diderot, D'Alambert y Voltaire, aprovechando el contexto Jean-Jacques Rousseau, escribe un trabajo importante en la época de la Ilustración titulada "el contrato Social", el cual desarrollo un esquema social, donde el poder recae sobre el pueblo y el tema de servicio público tiene relación con los intereses colectivos del Estado, es así como el autor tiene una visión de este conjunto de bienes y actividades que considera una noción política, y lo proyecta hacia objetivos de actividades estatales como las finanzas públicas y el ejército, dejando un poco de lado la visión solo jurídica. (Rozas, y Hantke, 2013).

\subsection{Noción del Servicio Público}

Cabe resaltar la contribución dada por las jurisprudencias del tribunal de conflictos y del Consejo de Estado, en el estudio y avance de la doctrina del servicio público, fallos 
Marta Lucía García González y Mario Armando Echeverria Acuña

jurisprudenciales como hitos en la formulación del concepto de servicio público, como el famoso fallo blanco de 1873, que consagra la responsabilidad del estado así de esta manera poniendo fin a una serie de irresponsabilidades y por otro lado reconoce el servicio público como elemento de competencia del Tribunal Administrativo.

En un segundo lugar el fallo Terrier 1903, afianzo aún más el concepto de servicio público, a lo local, al establecer que todo lo relacionado con el mismo respecto a su organización y sobre todo su funcionamiento se debe a la jurisdicción administrativa, de esta forma arraigo la unificación del contencioso de las entidades territoriales con el estado. (Romero, 2015). Posteriormente al Concejo de Estado Frances se le unió otra decisión, el Fallo Vosges 1912, por medio del cual se definió las cláusulas exorbitantes del derecho común en los contratos administrativos de los servicios públicos, con el fin de delimitar competencias. (Romero. 2015).

Sobre toda esta realidad jurisprudencial llega la postura del jurista francés, León Duguit, con la noción de servicio público la cual fue desarrollada por León Duguit junto con Gastón Jeze, bajo una óptica social, totalmente separadas de los pensamientos individualizados que caracterizaba aquella época de la Revolución Francesa. Para León Duguit, la noción del servicio público se convirtió en noción fundamental de derecho público moderno. (Mercado, 2015),

\section{"todo el problema de la actividad del Estado se resuelve en esta antítesis: mandar o servir",}

al lado de esta concepción sostuvo que servicios modernos que en esos momentos existían como correos, alumbrado, teléfonos, ferrocarriles y obras públicas, donde el Estado intervenía y debía ser regulada por el Derecho Público. (Rebollo, 1983).

Además, Duguit mencionaba que debido a la importancia que tiene la actividad de los servicios públicos y su relación con la población debe ser una actividad no solamente asegurada sino también regulada y controlada, de esta manera limita el poder de la autoridad, en este orden de ideas se puede inferir que la noción de servicio público evolucionaba dejando de ser solo un concepto estrictamente jurisprudencial y transformándose en un concepto ideológico. (Colín, 2008,). Se debe agregar, que la teoría de los servicios es esencial para hablar de Estado en la búsqueda de un interés general al servicio de la colectividad (Maldonado, 2010).

\subsection{Colombia y los servicios públicos}

La doctrina del servicio público nace y se desarrolla en Francia, doctrinas que cruzan fronteras, de ahí que muchos países adoptaron este sistema del Derecho Administrativo 
tales como: Suecia, Finlandia, Uruguay, Alemania, Italia, Bélgica, Colombia, los países bajos, Turquía. En el caso de Colombia, este adopto en su Constitución Política de 1991, el texto fundamental de la obligación de la prestación de los servicios esenciales por parte del Estado, servicios que pueden ser prestados por el mismo estado o por los particulares y bajo la vigilancia, supervisión del Estado Colombiano tal como se contempla en el artículo 365 de la Carta Magna, en (Salomoni, 1999 citado en Echeverry, 2013 p. 115) se señala que "cuando existe una necesidad que exceda a los individuos [esto es, que estos no pueden satisfacer por sí mismos], esa necesidad es pública [y por lo mismo de interés general]y siempre que esa necesidad sea de tal naturaleza, el Estado tiene la potestad y por lo tanto el deber de intervenir para asegurar su cumplimiento", todo lo anterior a través de sus actividades estatales, ya sea delegando o mediante concesiones, ya sea por particulares o directamente por el mismo, como finalidad del Estado Social de Derecho, teniendo los servicios público, el estatus constitucional e inherentes a la finalidad social del Estado.

\subsection{Conceptualización de Servicio Público de Transporte Urbano de Pasajeros en Colombia}

Como se había mencionado antes con respecto a la relación que existe entre los servicios públicos y su población, lo dicho por Duguit, que esta actividad debía ser garantizada, controlada y regulada por el Estado, de esta manera el transporte público urbano en Colombia, tiende a ser un servicio público con esas características, tal prestación es manejada por operadores privados, sin embargo, la infraestructura para su funcionamiento corresponde al estado.

El artículo 3 de la Ley 105 de 1993, indica que como personas que hacemos parte de una sociedad tenemos derecho a movilizarnos como pasajeros en vehículos que nos garanticen no solo calidad, sino también seguridad, prestación regulada por la ley, expedida por el congreso de la república, por expreso mandato de la carta política en el artículo 150, numeral 23; ahora frente a su operabilidad el transporte público como industria puede ser suministrado por empresas privadas, sin dejar de lado el interés general que está por encima del interés particular. Otro punto es la Ley 336 de 1996, refuerza aún más la responsabilidad que tienen las empresas de transporte público frente al servicio prestado y protección del usuario, bajo la supervisión del Estado, quien ejercerá el control y la vigilancia, bajo los términos de los artículos 333 y 334 de la Carta Magna.

El servicio de transporte público está íntimamente unido al derecho de libertad de locomoción, como se ha observado a lo largo de la jurisprudencia, a si pues la Sentencia T604 de 1992, sostiene que el servicio público de transporte prestado de manera intermitente contribuye a la amenaza de derechos como la libre circulación, el estudio, el trabajo, la recreación, entre otros. (Gómez, 2011). Es por ello en virtud de la ley, el transporte público 
Marta Lucía García González y Mario Armando Echeverria Acuña

es señalado como un servicio esencial, lo que hace que tenga una relación de conexidad con los derechos fundamentales.

\subsection{El Sistema Integrado de Transporte Masivo, como una Política Pública en Colombia}

Para empezar hacemos un poco de historia mencionando que el primer sistema de transporte público masivo que hubo en Colombia, surge en Bogotá a mediados del siglo XX, el tranvía, y luego se extendió a otras ciudades como Barranquilla, Cúcuta, Pereira, Girardot, en Bogotá, fue administrado por la Empresa de Tranvía de Bogotá, servicio que transportaba 163 pasajeros diariamente, inaugurado en 1884 , la primera línea fue entre el puente de San Francisco y el Caserío de Chapinero, el pasaje costaba \$0,05, para los obreros que salían a trabajar en la madrugada era de \$0.02, para estudiantes menores de 14 años la tarifa se reducía al 50\% sistema que prestó sus servicio por 65 años, hasta 1923 que empezó a circular los buses de gasolina, particulares y del municipio.(Triana,1996).

Así pues, se permite demostrar que los megaproyectos de sistemas de transporte público masivo, también existieron hace cien años, en Bogotá y otras ciudades, como estrategias que daban respuesta a las necesidades del transporte urbano, los cuales cambian a medida que apuntan otros aspectos como el incremento de la población, la evolución de las ciudades que no son distintas a las de hoy en día. (Cárdenas, 2011).

Por lo que se refiere a las primeras políticas públicas de transporte masivo, en primera instancia encontramos el Consejo Nacional de Política Económica y Social (CONPES), como máxima autoridad nacional de planeación en el desarrollo económico del país, emite el CONPES 3167 del 2002, política orientada a mejorar el transporte público urbano de pasajeros, con el diagnóstico del deterioro de la calidad del servicio que se venía prestando en las últimas décadas y concibiendo el transporte masivo como una suma entre equipo e infraestructura.

Luego se expide el CONPES 3259 del 2003, con la finalidad de avalar no solo técnicamente sino también financieramente el megaproyecto del Sistema Integrado de Transporte masivo a la ciudad de Cartagena, con el antecedente de la tasa de crecimiento poblacional la cual era de12,2\%, considerada una de las más altas del país.

En este orden de ideas se expide el documento CONPES 3260 de 2003, le da un impulso a las grandes ciudades del país para que se implementen los megaproyectos de los sistemas integrados de transporte masivo, con la meta de mejorar la calidad de vida y desarrollo urbano. Es así como se implementa este modelo de gestión urbana en las diferentes ciudades como: Pereira llamado (Megabús), en Cartagena denominado, (Transcaribe), en 
Bucaramanga recibió el nombre de (Metrolínea), en Medellín mencionado como (Metroplús), en Barranquilla (Transmetro) y por último en Cali (MIO).

Dentro de los lineamientos o conjuntos de acciones adoptadas por el Conpes 3305 del 23 de agosto de 2004, para la política del desarrollo urbano del Gobierno nacional, se obtuvo una óptica del crecimiento de la ciudad de Cartagena, su movilidad, sus necesidades, sus servicios públicos, su pobreza y el transporte urbano de pasajeros, con los lineamientos del plan de ordenamiento territorial (POT), nos permite analizar que congestiones en ese momento presentaba la ciudad de Cartagena a nivel urbano. Estos lineamientos son pertinentes en nuestra investigación porque permite analizar de qué manera fortaleció el transporte formal la calidad de vida de los Cartageneros, para tener un referente sobre la manera como podría mejorar el transporte en la ruta Daniel Lemaitre y Torices.

La gobernabilidad de la política pública de TRANSCARIBE en la ciudad de Cartagena se apoya jurídicamente en la siguiente normatividad: Ley 336 de 1996, se refiere al estatuto Nacional de Transporte, en su artículo 85, el cual indica que, a través de aporte sociales, dinero o especie puede participar la Nación al igual que las entidades descentralizadas, en la solución de estos megaproyectos de transporte masivo.

Además, para estos megaproyectos de Servicio público urbano, tanto el Estado Colombiano como Nación y sus entidades descentralizadas por servicios, juegan papeles importantes donde pueden participar con un $40 \%$ como mínimo en aportes de capital y un $70 \%$ como máximo, interpretado por la ley 310 del 6 de agosto de 1996 en su artículo 2; Sin dejar atrás el Decreto 3109 de 1997 el cual reza sobre la reglamentación de la habilitación y prestación del servicio masivo de pasajeros, así mismo la manera en que se utilizarían los recursos de la nación, y por último la Resolución 230 del 19 de diciembre de 2014 donde se consigna el documento del Plan Estratégico 2013-2018 que da entrada al Sistema de Transcaribe S.A.

\subsection{TRAnSCARIBE y la gobernabilidad de una Política Pública de Transporte Masivo en Cartagena}

Guarín (2003) nos muestra a la Cartagena no solo como la ciudad turística por sus encantos, también nos la visualiza como la Cartagena oculta, con problemas sociales, políticos y económicos que reflejan la informalidad de la ciudad, cuando se excluyen sectores, circunstancia que repercute por mencionar uno, el acceso al suelo urbano a la hora de adquirir una vivienda a través de programas institucionalizado; se puede observar el análisis realizado sobre la dimensión social del Sistema Integrado de Transporte, que impacta en lo urbano cuando se habla de invasiones que no deja de ser la manera común de ocupar espacios por los asentamientos de la población cartagenera, y esta es otra temática que se 
Marta Lucía García González y Mario Armando Echeverria Acuña

aborda desde el servicio de transporte público, punto importante porque repercute en la investigación que se aborda sobre la informalidad de los mismos al tratarse de la movilidad informal que padecen los habitantes de las invasiones porque el servicio formal de transporte no llega sino solo a los barrios vecinos.

Transcaribe S.A. ha habilitado varias rutas como por ejemplo la de Bocagrande, Gobernación de Bolívar, Crespo, Blaz de Lezo, como también la Variante llamada ruta de estudiantes, la cual beneficia la zona escolar tales como el Sena y la Universidad Tecnológica y la Universidad San Buenaventura, El Pozón, Nelson mándela, entre otras.

La empresa Transcaribe S.A, en aras de seguir mejorando la calidad de movilidad en la ciudad, impulso otra nueva ruta expresa, cuyo trayecto es desde el Patio Portal hasta la ciudad amurallada, recorrido que hace por toda la troncal del sistema, es decir por la avenida Pedro Heredia, durante 15 minutos. Según lo anterior podemos observar que a través de una política pública de movilidad en el trasporte urbano de pasajeros, la cual se interpreta como la capacidad que tiene el estado de crear estrategias que canalicen las demandas sociales que brinden soluciones adecuadas a las mismas, que permitan balancear las relaciones que debe existir entre gobierno-ciudadanos, que no deja de ser el resultado, de un sistema político que en Colombia se basa en el principio medular: el Estado Social de Derecho, considerado democrático y acoplado al POT, como el instrumento básico para desarrollar el proceso de ordenamiento del territorio municipal o Distrital, apoyado por el Decreto 0977 de 2001, teniendo en cuenta que es el conjunto de las diferentes directrices, al igual que políticas, como también de estrategias que van dirigidas a distintas normas y programas, que debe tener en cuenta los municipios con la finalidad de orientar y administrar el desarrollo físico del territorio y la utilización del suelo, como herramienta jurídica, para discutir temas relacionados con el aspecto urbano y el espacio público.

\subsection{El transporte informal frente a una política pública de movilidad insuficiente}

Al principio de esta investigación se mencionó de acuerdo al Decreto No. 0067 de 2008, emanado por el (DATT), la restructuración en las rutas de los buses que prestaban el servicios de transporte en el barrio Daniel Lemaitre hacia el Centro de la ciudad y viceversa, las cuales fueron concedidas las empresas RENACIENTE (Con 19 buses), RODRÍGUEZ TORICES (Con 18 buses), ETUL (Con 6 buses), Cootransbol que luego se convirtió en VEHITRANS (Con 6 buses), FLOTA DE LUJO (Con 4 buses), MEDIA LUNA (Con 4 buses) y PEMAPE (Con 3 buses) para un total de sesenta (60) buses urbanos, de manera transitoria hasta la implementación del sistema masivo de transporte.

Aunado a esto, el Plan de Desarrollo "Ahora si Cartagena", 2013-2015, sentó las bases para atender las necesidades insatisfechas de los habitantes de Daniel Lemaitre, San Francisco, 
La María, entre otras, con respecto a la falta de rutas, teniendo en cuenta que de las 45 rutas que hasta ese entonces estaban contempladas, solo funcionaban 27 y cubrían solo la mitad de los barrios de la ciudad, de esta manera se veía afectado el 50\% de la población sin servicio de transporte. En ese mismo contexto solo funcionaban 1.300 buses y busetas de los 1.591 que había autorizado el DATT, el resto de buses se encontraban embargados o en su defecto dañados, es así como resultado de estos percances empieza la mala prestación del servicio y como consecuencia la población acude a otras alternativas a través del transporte informal e ilegal (jeeps colectivos, mototaxis).

Cuando se habla de movilidad no solo se hace mención del significado de desplazarse de un lugar a otro sino también involucra a un contexto social y cultural referente a ese desplazamiento, respuesta a ese entorno social y económico que envuelve dicha circunstancia. Por otro lado cuando una política pública, no alcanza a cubrir las necesidades de transporte a medida que crecen las ciudades, especialmente aquellas que se expanden en la periferia que por lo general habitan personas con ingresos demasiados bajos, da lugar a que la misma población busque otras alternativas que suplan sus necesidades de movilidad no autorizados legalmente, como el transporte informal, que se resume en al transporte público terrestre individual de pasajeros por fuera de la ley, utilizando vehículos que no cumplen con la tecno-mecánica reglamentada por el Código de Transito de Colombia.

Para Thomson (1993), los bajos recursos con que cuentan los habitantes de zonas marginales no es el único obstáculo, para no tener acceso a un transporte público urbano, a este se asocian problemas internos y externos del mismo, por consiguiente los factores internos hace mención a la manera como se planifica y administra el sistema de transporte, en esta categoría se encuentra la baja proporción de vehículos que salen a prestar el servicio público, la reglamentación excesiva con la que deben cumplir los vehículos hace que estos se limiten a prestar el servicio en el sector, además el gasto público que desembolsa el sector público suele invertirse más en metros y vías rápidas que en el trasporte urbano, situación que no beneficia a los hogares más pobres. De igual importancia encontramos los factores externos al que se enfrentan los administradores de las empresas de transporte urbano tales como: el transporte urbano no puede en muchas ocasiones acceder a las zonas urbanas donde habitan poblaciones de extrema pobreza debido al mal estado de los terrenos, además la política económica influye en el transporte urbano, por lo tanto en muchas ocasiones sus intereses no están centrados en el sector transporte a no ser que se presente alguna crisis de interés.

Las políticas públicas orientadas a la movilidad, si bien es cierto dentro de sus prioridades es solucionar las necesidades de la población referente a la movilidad, dentro de esta planeación es pertinente analizar las características socioeconómicas de la población, 
Marta Lucía García González y Mario Armando Echeverria Acuña

información de gran importancia que arroja un diagnostico que permite plantear el transporte en ese entorno, es decir para justificar la implementación de una ruta se debe examinar la demanda actual y futura del sistema, con el fin de proporcionar un servicio eficiente y sobre todo económico. (Posada y González, 2010).

\subsection{Los derechos económicos sociales y culturales de los propietarios y conductores vinculados a la Cooperativa Multiactiva de Transporte Colectivo, Comutrascol en la ruta Daniel Lemaitre, en la ciudad de Cartagena y su afectación por la implementación de TRANSCARIBE}

Si bien es cierto los Derechos Económicos, sociales y culturales son los derechos que le permiten a todo individuo gozar de una calidad de vida digna, según el artículo 25 de la Declaración Universal de los Derechos Humanos:

"Toda persona tiene derecho a un nivel de vida adecuado que le asegure, así como a su familia, la salud y el bienestar, y en especial la alimentación, el vestido, la vivienda...".

De igual manera el Comité de derechos Económicos, Sociales y Culturales de las Naciones Unidas (CDESC), observa el efectivo cumplimiento por parte de los estados que han ratificado el PIDESC.

En relación con el tema anterior al eliminar los camperos que hacen de Colectivo en la Ruta Daniel Lemaitre para darle paso a los vehículos de Transcaribe pueden presentarse afectaciones de tipo socio-económico en las familias de los propietarios y conductores que están integrados en la Cooperativa Multiactiva de Transporte Colectivo, Comutrascol, ya que afectaría sus necesidades básicas como la vivienda, la educación de sus hijos, la alimentación, por lo que el Distrito debe estudiar la posibilidad de establecer planes de acción o políticas públicas con un fuerte contenido en derechos humanos, que se implementen con el gremio de los camperos antes de sacarlos del servicio, ellos quieren charratización, indemnización y que se les incluya como trabajadores de Transcaribe.

El Ministerio de Transportes alega que no se puede dialogar con ellos, porque son ilegales y la ex alcaldesa Yolanda Wong fue una de las personas que impulsaron el estudio socioeconómico que hizo el Colegio Mayor de Bolívar, el cual arrojó como resultado el perjuicio que Transcaribe causaría a los colectivos. Los integrantes de la Cooperativa Multiactiva de Transporte Colectivo, Comutrascol aceptan que deben salir, pero con las garantías laborales que les permita conseguir su sustento cotidiano, porque tienen más de 25 años prestando el servicio de transporte, aunque se les diga que es ilegal, ellos alegan que no es ilegal, es informal, al igual exigen no se les vulnere el derecho al trabajo y mínimo vital, además preguntan porque no han sido incluidos en la política social de Transcaribe, 
situación que han informado ante el Honorable Concejo Distrital de Cartagena por intermedio de su representante Oswaldo Pérez Ávila, en la invitación a un debate de control político sobre movilidad, tránsito y Transcaribe, en sesión 21 de marzo del 2018, donde asistieron: el gerente de Transcaribe, la Secretaria de Infraestructura, el Secretario de Planeación, además estuvieron presentes el Contralor (e) y el Personero Distrital.

Se hace necesario resaltar que en Documento Conpes 3260 de 2003, se le solicito al Distrito de Cartagena que a través del SENA se crearan capacitaciones laborales dirigidas a los transportadores que actualmente se encontraban vinculados al sistema, que resultaran afectados por la implementación del SITM.

El presente estudio toca con los derechos económicos, sociales y culturales, (Castro, Crespo y García, 2009) en este sentido señalan que, en un Estado Social de Derecho, estos tienden a tener suma importancia a la hora de concebir los fines del estado que concentran una serie de intereses como la dignidad humana, justicia social y libertad económica en el contexto de un estado regulador.

Colombia a través de la carta política de 1991, se define como un Estado Social de Derecho, acreditando una relevancia particular a los derechos humanos, que permite anclarlos derechos económicos, sociales y culturales, al igual que los políticos y civiles. Alcalá, (2009) señala que estos derechos están revestidos con valores de dignidad, igualdad, enfocados a traspasar las desigualdades sociales, como un mínimo vital que se ajuste a la dignidad humana de un individuo, a través del derecho y prestaciones que de manera directa o indirecta pueden ser ofrecidos por el estado.

Ahora referente a la justiciabilidad de los DESC, la Corte constitucional ha manifestado que, aunque estos no estén considerados como derechos fundamentales, si pueden ser requeridos mediante el ejercicio de la acción de tutela para lograr su garantía siempre y cuando cumpla con el criterio de conexidad con derechos que ostenten tal naturaleza. (Cortes et al., 2007)

En otras palabras en la sentenciaT-772 de 2003, se interpreta que la ejecución de algunas políticas, desafortunadamente se convierten en fuente de pobreza para los afectados, en el sentido de desconocer el deber estatal de suprimir contextos hostiles que generan condiciones frágiles en la población, políticas que no cuentan con mecanismos complementarios con el fin de apaciguar de manera proporcional los efectos negativos que pueda ocasionar, situación injusta frente a los derechos económicos, sociales y culturales, resultando desproporcional al principio de Estado Social de Derecho. 
Marta Lucía García González y Mario Armando Echeverria Acuña

En la medida que las políticas públicas debe partir de un estudio eficiente de la verdadera realidad de una población, por medio de los estudios técnicos que permita en lo posible observar el alcance negativo que la estrategia de la política pueda ocasionar, convirtiéndose en una carga publica desproporcionada que no deben soportar, aun cuando se encuentra en condiciones vulnerables a causa de la pobreza, en la ejecución de la política debe actuar paralelamente la medida necesaria para reducir el daño recibido, con el fin de respetar el derecho al mínimo vital y por ende la subsistencia en condiciones dignas. (Sentencia T$772,2003)$

Dentro de este orden de ideas en la sentencia T-348 de 2010, brevemente se trata de un grupo de pescadores (ASOPESCOMFE), quienes instauraron acción de tutela contra el Distrito turístico de Cartagena, INCO, la DIMAR y el Ministerio de Ambiente y Desarrollo Sostenible, ya que había vulnerado el derecho al trabajo, a la libre escogencia de profesión, vida digna entre otros, por no garantizarles una consulta previa, en la ejecución de un proyecto de infraestructura en el Anillo vial malecón de Cartagena, alegando que ejercían la pesca artesanal por más de 50 años. Así mismo dentro de la consideraciones la corte advirtió teniendo como referencia la sentencia T-557 de 2006, que pese a que el derecho al trabajo es un derecho catalogado dentro de los DESC, por su naturaleza prestacional y progresividad, sin embargo esta reevaluado el criterio y considera que esta característica también es de los derechos civiles y políticos, así como lo es deber de abstención aplicable a los DESC., lo que significa que la distinción entre unos y otros no es criterio suficiente para negar el tratamiento como derechos fundamentales a los derechos sociales, en este caso al derecho al trabajo.

\section{CONCLUSIÓN}

Actualmente las colectividades locales y regionales buscan la inclusión de los actores sociales en el desarrollo de las políticas públicas, de acuerdo con los principios de la Reforma del Estado colombiano a partir de la Constitución Política de 1991, que fundamenta un Estado Social de Derecho en su Artículo $1^{\circ}$ y en el $2^{\circ}$ establece los fines del Estado, lo cual permite integrar los actores sociales a las respuestas gubernamentales. En ese contexto los planes de acción o estrategias políticas deben resultar de un acuerdo entre el Estado y la participación ciudadana, enmarcadas en política públicas con un alto contenido de Derechos Humanos, que se materialice a través de los estudios técnicos previos que puedan analizar con vigor las características socioeconómicas de la población, que permita llevar a cabo el proceso de integración para el eficaz ejercicio de la gobernabilidad, acompañado de programas que mitiguen los impactos negativos que la implementación de una política pública pueda generar en un contexto social. Como indica (Martínez y Marín, 2015) cuando el estado tergiversa las políticas públicas en estos casos se permite un desconocimiento constitucional de los DESC, así de esta manera penetrando 
en el contexto de los grupos más pobres lo cual implica una insatisfacción en esas poblaciones hasta el punto de no querer participar políticamente, cuando observan que sus necesidades no tienen un cambio social.

Por ello el análisis del sondeo estadístico realizado en grupos focalizados de la población que habita en la ruta Daniel Lemaitre, que utiliza el transporte informal de los colectivos Jeeps y Nissan, a los cuales se les aplicó una encuesta que se elaboró por parte de la investigadora, nos permite inferir que los ciudadanos cartageneros que habitan en los barrios por donde pasa la ruta de Transcaribe, piensan que cuando este Sistema Integrado de Transporte Masivo empiece a funcionar se verán vulnerados los Derechos Económicos, Sociales y Culturales de los propietarios y conductores de los camperos que prestan, aunque de manera informal, el servicio de transporte público, y el Distrito de Cartagena no tendrá en cuenta, vulnerándose además el principio de la confianza legítima por los años que tienen estas personas de hacer esta labor.

Se solicitó a través de un Derecho de Petición que se envió al Director de TRANSCARIBE, con radicado interno No. 2380 del 16 de agosto de 2019, que fue respondido con el oficio TC-DO-07.01- 1436-2019, en el que explica que sucedía con la ruta Daniel Lemaitre en el Sistema Integrado de Transporte aduciendo, que Dentro del diseño operacional del sistema integrado de transporte masivo de Cartagena, Transcaribe se tiene contemplado para estos sectores de la ruta mencionada la operación de una ruta auxiliar o complementaria, la cual tiene previsto operar con vehículos tipo Busetón con capacidad para 50 pasajeros.

Reconoce que las necesidades de transporte en la zona han sido suplidas para la población por un transporte informal, el cual es prestado por vehículos que no son homologables a la tipología de buses utilizada por el sistema integrado de Transporte Masivo. En este sentido Transcaribe no puede realizar su tratamiento como bien lo hace con los buses del TPC que legalmente se encuentran censados por la autoridad competente y se encuentran dentro de los listados del parque automotor oficiales para la respectiva cancelación de ruta, negociación y chatarrización y que ante las falencias del sistema de transporte público, el Gobierno crea estrategias a través de políticas públicas en la implementación de sistemas de transportes que mitiguen las diferentes necesidades que la población estaba presentando en materia de movilidad, bajo los criterios de eficiencia operativa, economía y ambiental, así de esta manera apoyando proyectos de transporte público fundados en utilización de vías exclusivas y buses de alta capacidad.

Finalmente la investigación muestra como TRANSCARIBE, no va a indemnizar a los dueños de los jeeps que fungen como colectivos en la Ruta Daniel Lemaitre cuando se implemente el Sistema Integrado de Transporte Masivo, lo que es posible que se torne como 
Marta Lucía García González y Mario Armando Echeverria Acuña

una situación de violencia contra los propietarios de los vehículos que son utilizados como colectivos, también los conductores de los mismos, los sparring, los controladores del tiempo, etc., todos los que viven del valor agregado del transporte público en la ruta mencionada en la ciudad de Cartagena.

Se concluye de lo anterior, que se denota una tensión social y jurídica entre el Distrito de Cartagena y TRANSCARIBE como operador del SITM, con los integrantes de la Cooperativa Multiactiva de Transporte Colectivo, Comutrascol entre otras, no solo por la violación de derechos fundamentales sino también por la posible violación del principio de confianza legítima, al cual la Corte Constitucional ha establecido los presupuestos que deben acreditarse: "La necesidad de preservar de manera perentoria el interés público; la demostración de que el particular ha desplegado su conducta conforme el principio de la buena fe; la desestabilización cierta, razonable y evidente en la relación entre la Administración y el particular y, finalmente; la obligación de adoptar medidas transitorias para que el particular se pueda acomodar a la nueva situación creada por el cambio intempestivo de actitud por parte de la administración". (Sentencia T-424 de 2017)

\section{REFERENCIAS BIBLIOGRAFÍCAS}

Alcreri, Vicente. (30 de noviembre de 2015) ¡Transcaribe tardó el mismo tiempo que gasta una nave en llegar a Marte! El Heraldo. Recuperado de El Heraldo. https://www.elheraldo.co/bolivar/transcaribe-tardo-el-mismo-tiempo-que-gasta-una-naveen-llegar-marte-230998

Berger, P. \& Luckmann T. (1998), La Construcción Social de la Realidad. Amorrortu Editores S.A. Buenos Aires. p.p. 1-115

Castro, E., Restrepo, O., \& García, L. (2009). Historia, concepto y estructura de los derechos económicos, sociales y culturales. Revista Estudios Socio-Jurídicos, Universidad del Rosario. No. 9, p.p. 77-108.

Caro Mejía, K. (2019) Hermenéutica judicial para la protección de los segundos ocupantes en el proceso de restitución y formalización de tierras. Revista Jurídica Mario Alario D'Filippo, Vol. 10, Número 19: 250-270.DOI: https://doi.org/10.32997/2256-2796-vol.10num.19-2018-2142

Consejo Nacional de Política Económica y Social. Documento Conpes 3305 de 2004: Lineamientos para optimizar la Política de Desarrollo Urbano. 
Corte Constitucional de Colombia, Sentencia T - 772 de 2003. (M. P. Manuel José Cepeda Espinoza). 4 de septiembre de 2003.

Corte Constitucional de Colombia, Sentencia T - 348 de 2010, (M. P. Jorge Ignacio Pretelt Chaljub) 11 de mayo de 2010.

Corte Constitucional de Colombia, Sentencia T - 424 de 2017. (M. P. Alejandro Linares Cantillo). 4 de julio de 2017.

Cortés, J., Arias, C., Fanger, N., González, A., Kurmen, A., Luna, B., Manrique, J., Prieto, E., \& Pulido, D. (2007). La naturaleza jurídica de los derechos económicos, sociales y culturales en la jurisprudencia de la Corte Constitucional. Revista Estudios Socio-Jurídicos. Universidad del Rosario. No. 9, p.p. 109-141.

Dangond, C., Jolly, J., Monteoliva, A. \& Rojas, F. (2011). Algunas reflexiones sobre la movilidad urbana en Colombia desde la perspectiva del desarrollo humano. Papel político, Vol. 16 No. 2, p.p. 485-514.

Decreto 243 de 1987. Por medio de la cual se le otorgó la habilitación, permiso o adjudicación de las rutas urbanas a la empresa FLOTA DE LUJO S. A. para la prestación del servicio de transporte público colectivo en la ciudad de Cartagena de Indias.

Decreto 0977 de 2001. Por medio del cual se adopta el Plan de Ordenamiento Territorial del Distrito Turístico y Cultural de Cartagena de Indias. 20 de noviembre de 2001.

Decreto 0667 de 2008. Por el cual se reestructura el recorrido y se hace una revisión integral del plan de rodamiento de las Rutas 1: ESPERANZA - CENTRO, Ruta 9: SANTA MARÍA - TORICES - CENTRO, Ruta 18: DANIEL LEMAITRE - CENTRO, Ruta 19: DANIEL LEMAITRE - MERCADO y Ruta 23: NARIÑO - CENTRO. Alcaldía Mayor de Cartagena de Indias D. T. y C. 27 de agosto de 2008.

Decreto 3109 de 1997. Por el cual se reglamenta la habilitación, la prestación del servicio público de transporte masivo de pasajeros y la utilización de los recursos de la Nación. 30 de diciembre de 1997.

Departamento Nacional de Planeación y Ministerio de Transporte. (2002) Política para mejorar el servicio de transporte Público urbano de pasajeros. Conpes 3167. Recuperado de https://colaboracion.dnp.gov.co/CDT/Conpes/Econ\%C3\%B3micos/3167.pdf 
Marta Lucía García González y Mario Armando Echeverria Acuña

Echeverri, Á. (2013). La noción del servicio público y el estado social de derecho. El caso colombiano. Novum Jus: Revista Especializada en Sociología Jurídica y Política; Universidad Católica de Colombia, Vol. 7, No. 2; p. 111-127.

Esquivel, R. (1996) Sociedad y transporte urbano en Bogotá 1865-1950. Memoria y sociedad, Vol. 1, No. 2, p.p. 19-37.

Gómez, C. (2011). Políticas de transporte urbano: El caso del sistema masivo de transporte en el área metropolitana de Cali. Revista de Economía \& Administración. Vol. 8; pp. 101123.

Gómez, O. (2011). Régimen jurídico del transporte terrestre en Colombia. Fondo de Prevención Vial. Bogotá.

Guarín, A., (2003). Cartagena de Indias: Asentamientos informales en la década de los 90. Revista Bitácora Urbano Territorial, Vol. 1, No. 7. P.p. 101-109.

Hampe, T. (1988). La Revolución Francesa vista por el Mercurio Peruano: cambio político vs. reformismo criollo. Boletín del Instituto Riva-Agüero, No. 15, p.p. 163-178.

Herreño, D. \& Fernández, L. (2008). Reflexiones sobre Gobernabilidad: el SITM como política pública. Santander-Colombia. Universidad Autónoma de Bucaramanga. Reflexión Política, vol. 10, núm. 19, p.p. 176-187.

Hurtado, A., Torres, A., \& Miranda, L. (2011). El programa de sistemas integrados de transporte masivo en Colombia: ¿un ejemplo de recentralización de la gestión de las ciudades. Revista Territorios, No. 25, p.p. 95-120.

Islas, A. (2008). El servicio público en el derecho francés. Actualidad de los servicios públicos en Iberoamérica. Editores, Universidad Autónoma de México, Instituto de Investigaciones Jurídicas, p.p. 277-292.

Ley 310 de 1996. Por medio de la cual se modifica la Ley 86 de 1989. 12 de agosto de 1996. D.O. No. 42.853.

Ley 336 de 1996. Estatuto General de Transporte. 20 de diciembre de 1996. D. O. No. 42.948 . 
Ley 688 de 2001. Por medio de la cual se crea el Fondo Nacional para la Reposición del Parque Automotor del Servicio Publico del Transporte Terrestre y se dictan otras disposiciones. 24 de agosto de 2001. D.O. No. 44.530.

Maldonado, T. (2010). La noción de servicio público a partir de la concepción del Estado Social de Derecho. Revista Actualidad Jurídica. P.p. 54-62.

Martín, L. (1983). De nuevo sobre el servicio público: planteamientos ideológicos y funcionalidad técnica. Revista de Administración Pública, No. 100-102, pp. 2471-2542.

Martínez, H., \& Marín, J. (2015). Protección de los derechos económicos, sociales y culturales en el ordenamiento jurídico colombiano - el papel de los jueces. Justicia Juris, Vol. 11, No. 1, p.p. 13-25.

Mercado, A. (2015). La influencia de León Duguit en la reforma social de 1936 en Colombia: El sistema jurídico, la función social de la propiedad y la teoría de los servicios públicos. Universidad del Rosario.

Nogueira, H. (2009). Los derechos económicos, sociales y culturales como derechos fundamentales efectivos en el constitucionalismo democrático latinoamericano. Estudios constitucionales, Vol.7, No.2, p.p. 143-205.

Plan de Desarrollo 2002-2006, "Hacia un Estado Comunitario", Álvaro Uribe Vélez.

Plan de Desarrollo 2013 - 2015, “Ahora Si Cartagena”, Dionisio Vélez Trujillo.

Ramírez, E. (2001). La Investigación Socio jurídica. Editorial Doctrina y Ley. Bogotá.

Resolución 230 de 2014. Por medio de la cual se adopta el Plan Estratégico 2013-2018 para Transcaribe S.A. 19 de diciembre de 2014

Romero-Pérez, J. (2015). La primera fase histórica del servicio público. Revista de Ciencias Jurídicas, No. 138.

Saldarriaga, A. (2007). Macroproyectos de movilidad urbana y la construcción de la ciudad. Bogotá.

Thomson, I. (1993). Cómo mejorar el transporte urbano de los pobres. Revista de la CEPAL, No. 49, p.p. 137-154. 
Marta Lucía García González y Mario Armando Echeverria Acuña

Viguri Perea, A. y Chiara Marullo, M. (2016) El derecho a un medio ambiente sano y la encrucijada de los alimentos transgénicos. Revista Jurídica Mario Alario D'Filippo, Vol. 8, Número 15: 100-111. DOI: https://doi.org/10.32997/2256-2796-vol.8-num.15-2016-1526 